\title{
Sequence Similarity
}

National Cancer Institute

\section{Source}

National Cancer Institute. Sequence Similarity. NCI Thesaurus. Code C45333.

The degree of similarity between the primary sequence of two biological

macromolecules. 